\title{
Obstetrics-based clinical immersion of a multinational team of biomedical engineering students in Ghana
}

\author{
Kathleen H. Sienko ${ }^{\mathrm{a}, \mathrm{b}, *}$, Elsie Effah Kaufmann ${ }^{\mathrm{c}}$, Moses E. Musaazi ${ }^{\mathrm{d}}$, Amir Sabet Sarvestani ${ }^{\mathrm{e}}$, Samuel Obed ${ }^{\mathrm{f}}$ \\ a Department of Mechanical Engineering, University of Michigan, Ann Arbor, USA \\ ${ }^{\mathrm{b}}$ Department of Biomedical Engineering, University of Michigan, Ann Arbor, USA \\ c Department of Biomedical Engineering, University of Ghana, Legon - Accra, Ghana \\ d Department of Electrical Engineering, Makerere University, Kampala, Uganda \\ e Design Science Program, University of Michigan, Ann Arbor, USA \\ ${ }^{\mathrm{f}}$ Department of Obstetrics and Gynecology, Korle Bu Teaching Hospital, Accra, Ghana
}

\section{A R T I C L E I N F O}

\section{Article history:}

Received 28 March 2014

Received in revised form 10 June 2014

Accepted 16 July 2014

\section{Keywords:}

Capacity building

Engineering education

Ghana

Implementation engineering

Maternal health

Medical device design

Low-resource countries

Among other obstacles, the continued lack of access to health technology, especially in low-resource countries, will contribute to many failing to meet Millennium Development Goal 5 by 2015 [1]. There is growing recognition among the global health community of the role that appropriate cost-effective health technologies can play in supporting healthcare providers' ability to deliver minimum standards of care [2,3].

Several universities in the USA have established programs to teach undergraduate engineering students, typically from high-resource settings, how to assess health needs on-site in low-resource countries and develop appropriate health technology solutions. The shortcomings of such programs include the involvement of a limited number of local stakeholders (predominantly, healthcare providers) and the failure of promising concepts developed in follow-on design courses to bridge the "valley of death" [4] and transition beyond the classroom. In addition, it is increasingly recognized that local engineering talent in lowresource settings must be further developed to increase the likelihood that local solutions will be generated, manufactured, adopted, and successfully implemented. This will require stronger and more effective

\footnotetext{
* Corresponding author at: University of Michigan, 2350 Hayward St, 1109 G.G. Brown, Ann Arbor, MI 48109, USA. Tel.: +1 734764 8308; fax: +1 7346473170.

E-mail address: sienko@umich.edu (K.H. Sienko).
}

partnerships among the international and local academic and clinical institutions involved.

To address these issues we developed and implemented a unique program for students from the University of Michigan, USA, the University of Ghana, and Makerere University, Uganda, to gain practical experience co-identifying unmet maternal health needs in Ghana in collaboration with multicultural and multidisciplinary stakeholders. Institutional Review Board approval was not obtained because the work described did not use human subjects; this paper provides a description of an intercultural clinical immersion experience.

During 2011, engineering students from the three universities (Fig. 1) participated in a joint month-long clinical immersion project in the Department of Obstetrics and Gynecology at Korle Bu Teaching Hospital in Accra, Ghana, featuring design ethnography techniques including observations and interviews, needs statement formulation, and needs prioritization and selection. The phases of the program and its timeline are given in Fig. 2.

In preparation for the clinical immersion experience, students completed obstetrics/gynecology and engineering design reading and written assignments to develop clinical literacy and context for the front-end design activities to be performed in Ghana. During the immersion experience, students attended morning meetings and completed observations and interviews with healthcare providers while rotating through the labor and delivery ward, elective and emergency surgery departments, prenatal care, mother and newborn care, family planning clinic, outpatient department, and gynecological complications units. Daily information sharing and event processing sessions were held to discuss observations and interview results, formulate and edit need statements, and identify follow-up activities. A faculty member associated with the program led experiential learning debriefings during the first week of the month-long immersion experience and then held semiweekly telecoms with the students to provide feedback on the need statements, needs filtering, and preliminary user requirements and engineering specifications for the selected design project topics. An instructional aide also remotely assessed the students' triweekly written project deliverables via an online file exchange system.

The needs assessment resulted in the identification of 85 needs that were subsequently classified into eight broad categories: diagnostic (13), preventative (8), assistive (24), communication and decision- 


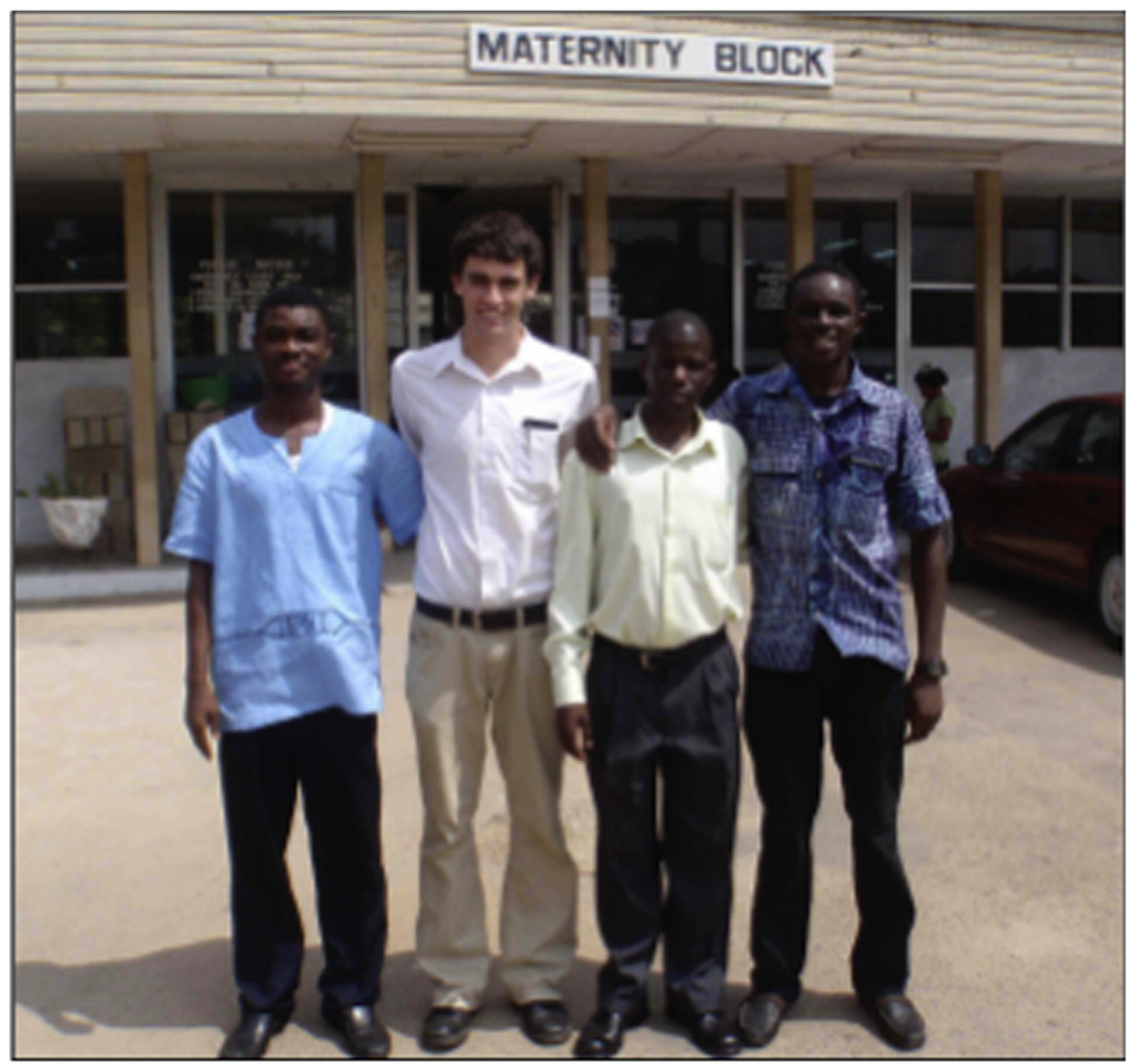

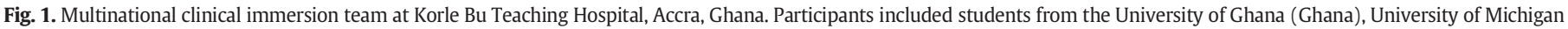
(USA), and Makerere University (Uganda).

making (8), organizational (15), sanitary (7), and supportive (10). Following the development and application of rubrics to identify and rank priorities, four distinct design project topics were selected in consultation with the department head and engineering faculty. Student projects stemming from the clinical immersion experience included a blood-warming device, assisted obstetric delivery device, adjustable labor and delivery bed, and a portable and attachable newborn cot.
Following the clinical immersion experience, the students returned to their home institutions and either completed their capstone design project or mentored a group of capstone design students on one of the four topics identified during the immersion experience. The pilot program outcomes included the generation of multiple prototypes, student-led design-based conference and journal publications, and peer-to-peer mentoring of non-immersion participants enrolled in the University of

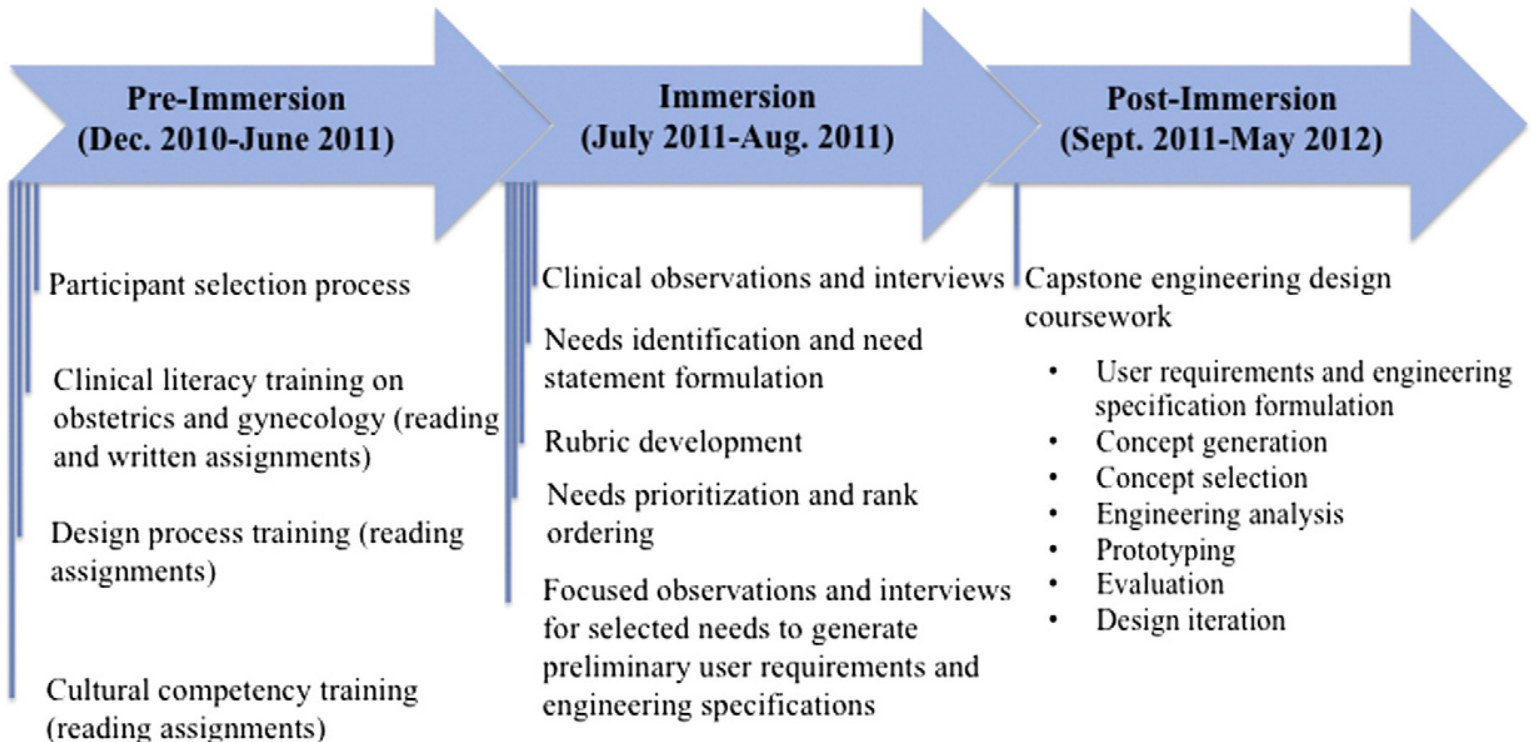

Fig. 2. Program phases and timeline. 
Ghana and University of Michigan capstone design courses by students who participated in the clinical immersion experience.

Programmatic challenges included coordinating academic calendars, recognizing and accommodating different academic program requirements, communicating the purpose of the experience within and outside of the multidisciplinary team, and managing expectations of participants and healthcare providers/stakeholders. Student challenges included accessing appropriate stakeholders, accessing online training materials, submitting program deliverables online, developing clinical literacy within the time constraints of the program, and developing user requirements and engineering specifications prior to the end of the immersion experience.

We believe that assembling a multinational engineering student team for this clinical immersion experience provided benefits for all partners: clinical and cultural immersion for the American students and an introduction to an innovative design culture for their African peers.

\section{Acknowledgments}

This material is based upon work supported by the University of Michigan African Studies Center, the National Collegiate Inventors and
Innovators Alliance (Program Grant \#7251-09), the National Science Foundation under Grant No. 0846471, the University of Michigan Multidisciplinary Design Minor: Specialization in Global Health Design, and the University of Michigan College of Engineering.

\section{Conflict of interest}

The authors have no conflicts of interest.

\section{References}

[1] Howitt P, Darzi A, Yang G-Z, Ashrafian H, Atun R, et al. Technologies for global health Lancet 2012;380(9840):507-35

[2] Spector JM, Reisman J, Lipsitz S, Desai P, Gawande AA. Access to essential technologies for safe childbirth: a survey of health workers in Africa and Asia. BMC Pregnancy Childbirth 2013;13:43.

[3] Free MJ. Achieving appropriate design and widespread use of health care technologies in the developing world. Overcoming obstacles that impede the adaptation and diffusion of priority technologies for primary health care. Int J Gynecol Obstet 2004; 85(Suppl. 1):S3-S13.

[4] Meslin EM, Blasimme A, Cambon-Thomsen A. Mapping the translational science policy 'valley of death.' Clin Trans Med 2013;2(1):14. 